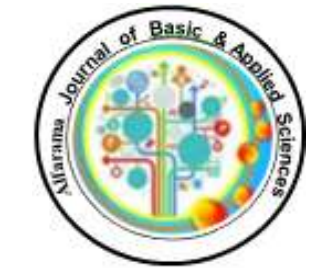

ISSN 2682-275X
Alfarama Journal of Basic \& Applied Sciences

Faculty of Science Port Said University

January 2021, Volume 2, Issue 1 https://ajbas.journals.ekb.eg

ajbas@sci.psu.edu.eg

http://sci.psu.edu.eg/en/

Submitted: 23-08-2020

Accepted: 04-10-2020

Pages: 123-134

\title{
Cellular and Simplicial Foldings of Surfaces
}

\author{
E. El-Kholy ${ }^{1}$, Nora. A. Omar ${ }^{2, *}$ \\ ${ }^{1}$ Mathematics Department, Faculty of Science, Tanta University, Tanta, Egypt \\ ${ }^{2}$ Mathematics and computer science Department, Faculty of Science, Port-Said University, Port-Said, \\ Egypt \\ *Corresponding author: alaa.nora99@yahoo.com
}

\begin{abstract}
In this paper, we develop the theory of cellular folding of compact connected surfaces onto polygons. The first question that naturally arises from the definition of cellular foldings are. "For a given compact connected surface $M$ and a given polygon $P_{n}$, is there any cellular folding of $M$ onto $P_{n}$ ?". Also if so, what are the possible topological types?. This is the existence problem.

Firstly, we discuss this problem and we obtain a wide range of existence theorems for cellular folding of a given surface onto a given polygon. Any simplicial folding decomposes the surface into simplexes of dimensions 0,1 and 2 which are called vertices, edges and faces respectively.

Secondly, we classify all the possible simplicial folding of the sphere, the connected sum of n-tori, and the connected sum of n-projective planes onto a polygon $P_{3}$. For each surface, we obtain certain relations satisfied by the number of vertices, edges, and faces of the simplicial decomposition of the surface to get either regular simplicial folding or just a simplicial folding.
\end{abstract}

\section{Keywords:}

Cell decomposition, Cellular folding, Folding graph, Simplicial folding.

\section{INTRODUCTION}

It is known that any surface $M$ is homeomorphic to exactly one of the following $S^{2}, T^{2} \# \ldots \# T^{2}$ ( $g$ factors) and $P^{2} \# \ldots \# P^{2}$ ( $p$ factors). Here $T^{2}$ and $P^{2}$ denote the torus and projective plane respectively, \# denote the connected sum operations and $g, p$ are natural numbers. The numbers $g, p$ are called the genus of $M$ and $S^{2}$ is said to have genus 0 . We denote by $M_{g}$ a closed orientable surface with genus $g$ and by $N_{p}$ a closed non-orientable surface with genus $p$. The surface homeomorphic to $S^{2}$ or $\#^{g} T^{2}$ are orientable with Euler characteristic $e(M)$ of $M$ equal to $2-2 g$, while those homeomorphic to $\#^{p} P^{2}$ are non-orientable with Euler characteristic $e(M)$ equal to $2-p$. 
Definition 1.1: A cell decomposition of a surface $M$ is a cell decomposition $C$ such that for each cell $\sigma$ of $C$, its closure $\bar{\sigma}$ is a closed cell, that is if $\sigma$ is an $n$-cell, $n=1,2$, then $\bar{\sigma}$ is homeomorphic to $D^{n}$. A closed 2cell is called a face of $C$, a closed 1 -cell is called an edge and a 0 -cell is called a vertex.

Definition 1.2 [1]: Let $M$ be a compact connected surface. A continuous map $f: M \rightarrow P_{n}$ of $M$ onto a polygon $P_{n}$ is called a cellular folding if there is a cell decomposition $C_{f}$ of $M$ such that:

1) $f$ is a cellular map of $C_{f}$ onto $C\left(P_{n}\right)$,

2) For each closed cell $\bar{\sigma}$ of $C_{f}$, the restriction map $f \mid \bar{\sigma}$ is a homeomorphism of $\bar{\sigma}$ onto a closed cell $\bar{\tau}$ of $C\left(P_{n}\right)$.

We call $C_{f}$ the cell decomposition of $M$ associated to $f$ or simply the cell decomposition of $f$.

Now, let $f: M \rightarrow P_{n}$ be a cellular folding and let $C_{f}$ be the cell decomposition of $f$, the edges and vertices of $C_{f}$ form a graph $\Gamma_{f}$ embedded in $M$. This graph is the singular point set of $f$ for which every vertex has even valency [2].

In this case the image $f\left(\Gamma_{f}\right)$ coincides with the boundary of $P_{n}$ and that the vertices of $\Gamma_{f}$ are sent to the vertices of $P_{n}$. The graph $\Gamma_{f}$ is called the folding graph. A regular folding is a cellular folding whose folding graph $\Gamma_{f}$ is regular. A regular folding $f: M \rightarrow P_{n}$ for which each vertex has valency $k$ is said to be a regular folding of type $(k, n)$.

A graph $\Gamma$ is n-colourable if and only if its vertices may be labeled with the numbers $1,2, \ldots, n$ in such a way that distinct end points of any edge have distinct labels. In the case of a graph $\Gamma$ embedded in a surface $M$, where each cell of $M \backslash \Gamma$ has $n$ vertices, we say that $\Gamma$ admits a cyclic n-colouring if it is n-colourable in such a way that the vertices of each cell are cyclically labeled in the sense that labels $1,2, \ldots, n$ are in their natural cyclic order module $n$.

The following propositions give us some characteristics of a finite connected graph $\Gamma$ embedded in a surface $M$ to be the edge graph of some cellular folding $f: M \rightarrow P_{n}$.

Proposition 1.3 [3]: Let $\Gamma$ be a finite connected graph embedded in a surface $M$, then $\Gamma=\Gamma_{f}$ for some cellular folding $f: M \rightarrow P_{n}$ if and only if $\Gamma$ admits a cyclic n-colorings.

Proposition 1.4 [3]: Let $f: M \rightarrow P_{n}$ be a cellular folding with $\alpha$ vertices, $\beta$ edges and $\gamma$ faces, then the following relations are satisfied:

1) $n \gamma=2 \beta$

2) $n \gamma \geq 4 \alpha$ (each vertex has valency $\geq 4$ )

3) Euler characteristic $e(M)=\alpha-\beta+\gamma \leq \alpha\left(\frac{4}{n}-1\right)$.

4) If $f$ is a regular folding of type $(k, n)$, then we have

$$
k \alpha=n \gamma=2 \beta
$$

5) If $M=M_{g}$ is an orientable surface with genus $g$, then we have

$$
g=1+\frac{(k-2)(n-2)-4}{4 n} \alpha
$$

6) If $M=N_{p}$ is non orientable surface with genus $p$, then

$$
p=2+\frac{(k-2)(n-2)-4}{2 n} \alpha
$$


Definition 1.5: Let $f: M \rightarrow P_{r}$ and $g: N \rightarrow P_{s}$ be cellular foldings, then we say that $f$ is equivalent to $g$ which is denoted by $f \approx g$ if and only if there are homeomorphisms $h_{1}: M \rightarrow N$ and $h_{2}: P_{r} \rightarrow P_{S}$ such that $g \circ h_{1}=h_{2} \circ f$.

It follows at once that $f \approx g$ if and only if there is a homeomorphism $h_{1}: M \rightarrow N$ such that $h_{1}\left(\Gamma_{f}\right)=\Gamma_{g}$. Hence $h_{1} \mid \Gamma_{f}$ is a graph isomorphism onto $\Gamma_{g}$. Also $f \approx g$ implies $r=s$.

Let $O_{g}(n)$ and $N_{p}(n)$ denote the set of equivalence classes of cellular $n$-foldings of an orientable surface of genus $g$ and a non-orientable surface of genus $p$.

As an immediate consequence of (3) in proposition 1.4, we have the following theorem.

Theorem 1.6 [4]: For each $n>4$, each $g \geq 0$ and each $p \geq 1$, the sets $O_{g}(n)$ and $N_{p}(n)$ are finite.

As an immediate consequence of (3) in proposition 1.4, we have the following theorems.

\section{Theorem 1.7 [4]:}

i. For any $n>3, O_{0}(n)$ and $N_{1}(n)$ are empty

ii. For any $n>4, O_{1}(n)$ and $N_{2}(n)$ are empty

Now, let $f: N_{p} \rightarrow P_{n}$ and $g: N_{q} \rightarrow P_{n}$ be cellular foldings. Choose two 2-cells $\sigma$ and $\tau$ of $N_{p}$ and $N_{q}$ respectively, let $A_{1}, A_{2}, \ldots, A_{n}$ be the vertices of $\sigma$ and $B_{1}, B_{2}, \ldots, B_{n}$ be the vertices of $\tau$ such that $f\left(A_{i}\right)=$ $g\left(B_{i}\right)$.

Remove the interior of $\sigma$ and $\tau$ from $N_{p}$ and $N_{q}$ respectively. Identify the resulting surfaces along the boundary of $\sigma$ and $\tau$ in such a way that $A_{i}$ and $B_{i}$ are obtained. This construction gives us the cellular folding of $N_{p} \# N_{q}$ to $P_{n}$, because the generated graph is also n-colourable with the colouring inherited from of $\Gamma_{f}$ and $\Gamma_{g}$.

\section{MORE RESULTS ON CELLULAR FOLDINGS}

Definition 2.1: We call the cellular folding obtained of $N_{p} \# N_{q}$ to $P_{n}$ a connected sum of cellular foldings $f$ and $g$ and is denoted by $f \# g$.

Theorem 2.2: Let $M$ be a closed connected surface, if there is a cellular folding $f: M \rightarrow P_{n}$ with $n=3$ or 4 , then there are infinitely many foldings of $M$ to $P_{3}$ or $P_{4}$.

Proof: If there is a folding $f_{1}: M \rightarrow P_{n}$, then according to proposition $1.3 \Gamma_{f_{1}}$ admits a cyclic $\mathrm{n}$-colouring.

For $n=3$, the graph $\Gamma_{f_{m}}$ obtained by taking the $(m-1)^{\text {th }}$ barycentric subdivision of $\Gamma_{f_{1}}$ is the graph of cellular folding $f_{m}: M \rightarrow P_{3}$, since $\Gamma_{f_{m}}$ admits a cyclic 3-colouring.

For $n=4$, since any square may be partitioned into $p q$ rectangles by a grid of lines parallel to the edges of the square for any given integers $p, q \geq 2$. The graph $\Gamma_{p q}$ obtained by this subdivision is the graph for a cellular folding $f_{p q}$ of $M$ to $P_{4}$, since $\Gamma_{f_{p q}}$ admits a cyclic 4-colouring.

Theorem 2.3: If there is a cellular folding $f: M_{g} \rightarrow P_{n}$, then there are infinitely many foldings of $M_{g}$ to $P_{3}$.

Proof: If there is a folding $f: M \rightarrow P_{3}$, then from theorem 2.2 there are infinitely many foldings of $M$ onto $P_{3}$. Consider the case where there is a folding $f: M \rightarrow P_{n}$ for $n>3$. Since there is always a folding $g \circ f P_{n}$ to $P_{3}$, we have the composition $g \circ f: M \rightarrow P_{3}$ which is a cellular folding. Then again from theorem 2.2 there are infinitely many foldings of $M$ to $P_{3}$.

Theorem 2.4: If there is a cellular folding $f: N_{p} \rightarrow P_{n}$ then there are infinitely many foldings of $N_{p}$ to $P_{3}$.

Proof: Is similar to the proof of theorem 2.3. 
It should be noted that the classes $O_{1}(3)$ and $O_{1}(4)$ both infinite [3]. Suppose that $M$ is an orientable surface of genus $g>1$, then $M$ is homeomorphic to $\#^{g} T^{2}$. We can exploit this fact together with the previous note to show that cellular foldings of $M$ to a triangle and to a square are plentiful.

Theorem 2.5 [3]: For all $g>1, O_{g}(3)$ and $O_{g}(4)$ are infinite.

Now, for a compact connected non-orientable surface $N_{p}=\#^{p} P^{2}$ without boundary of genus $g \geq 1$, $p \geq 1$, there is a double covering $\theta_{p}: M_{p-1} \rightarrow N_{p}$, where $M_{g}=\#^{g} T^{2}$. Thus, if $f: N_{p} \rightarrow P_{n}$ is a cellular folding, then so is $f \circ \theta_{p}=f^{*}: M_{p-1} \rightarrow P_{n}$. Conversely, any cellular folding $\varphi: M_{p-1} \rightarrow P_{n}$ that is equivalent under the covering group of $\theta_{p}$ induces a cellular folding of $N_{p}$ to $P_{n}$.

Using these facts, we can deduce the following results for non-orientable surfaces from those obtained for the orientable case.

Theorem 2.6 [3]: $N_{1}(3), N_{2}(3)$ and $N_{2}(4)$ are infinite.

Theorem 2.7 [3]: For all $p \geq 1, N_{2 p}(3)$ and $N_{2 p}(4)$ are both infinite.

This theorem can be proved by the use of the connected sum operation.

\section{Theorem 2.8:}

i. $\quad$ For all $p \geq 1, N_{p}(3)$ is infinite.

ii. For all $p \geq 2, N_{p}(4)$ is infinite.

Proof: The proof follows by using connected sum operation. To prove infiniteness of $N_{p}(3)$, we know from theorem 2.6 that both $N_{1}(3)$ and $N_{2}(3)$ are infinite. From theorem 2.7 we know that $N_{2 p}(3)$ is infinite for all $p \geq 1$. Again using the connected sum of both $N_{1}(3)$ and $N_{2 p}(3)$ it follows that $N_{2 p+1}(3)$ is infinite for all $p \geq 1$. Hence $N_{p}(3)$ is infinite for all $p \geq 1$.

To prove infiniteness of $N_{p}(4)$, we know from theorem 2.7 that $N_{2 p}(3)$ is infinite for $p \geq 1$. If we know that $N_{3}(4)$ is non-empty, then using theorem 2.2 we know that $N_{3}(4)$ is infinite. Then, again by theorem 2.2 and by using the connected sum operation for an element of $N_{3}(4)$ and elements of $N_{2 p}(4)$, we get that $N_{2 p+3}(4)$ is infinite.

The following is an example of folding $N_{3}$ to $P_{4}$,

Example 2.9: The cellular folding $f: N_{3} \rightarrow P_{4}$ is constructed by the topological identifications of the edges as shown in Fig. 1
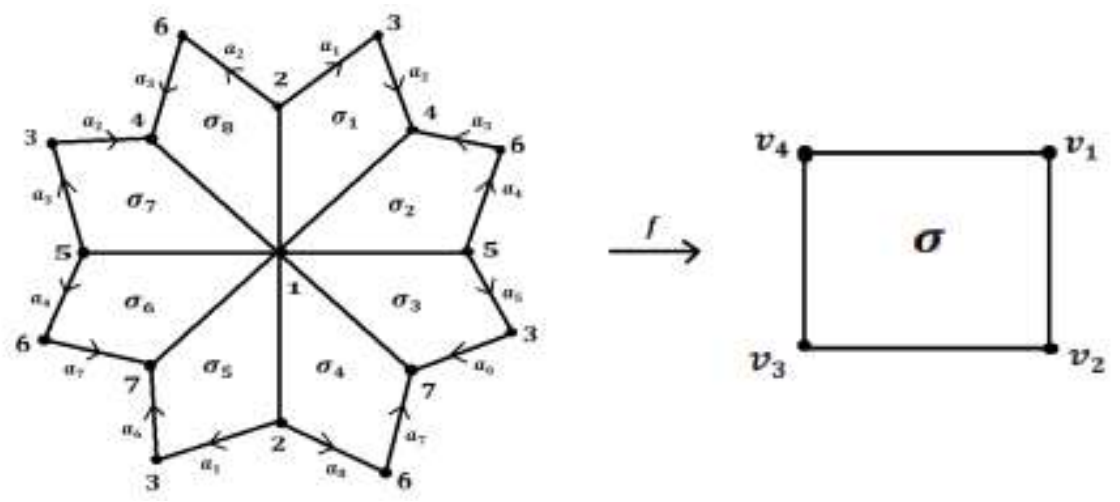

Fig. 1 Cellular folding from $N_{3}$ to $P_{4}$

In Fig. 1, the vertices are labeled 1,2, ., 7. The edges are identified according to the letters and orientation. 


\section{SIMPLICIAL FOLDING OF ORIENTABLE SURFACES}

The study of folding of complexes began with E. El-kholy and M. El-Ghoul [5] which was motivated later in [6].

Definition 3.1 [2]: Let $M$ and $N$ be simplicial complexes, a simplicial map $f: M^{(0)} \rightarrow N^{(0)}$ from $M$ to $N$ is a simplicial folding if for every $i$ and all $\sigma \in M^{(i)}, f(\sigma) \in N^{(i)}$ i.e., $f$ maps $i$-simplexes of $M$ to $i$ simplexes of $N$

In the following subsections, we classify all possible foldings of the sphere and the connected sum of n-tori onto a polygon $P_{3}$.

\subsection{Simplicial folding of the sphere}

The map $f: S^{2} \rightarrow P_{3}$ given by $f(x, y, z)=(|x|,|y|,|z|)$, where $S^{2}: x^{2}+y^{2}+z^{2}=1$ is the unit sphere in Euclidean space $E^{3}$ and $P_{3}$ is the intersection of $S^{2}$ with the positive octant, is a simplicial folding. In this case, the graph $\Gamma_{f}$ is isomorphic to the edge graph of the octahedron and it is called octahedral folding $\omega$ of $S^{2}$, see Fig. 2 .

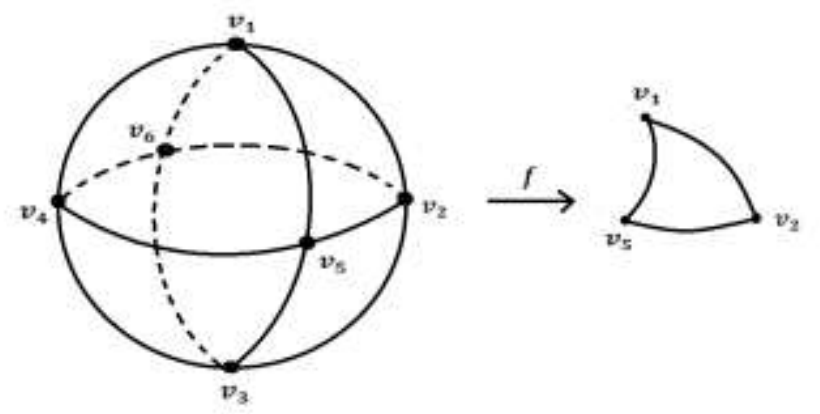

Fig. 2 The octahedral folding of the sphere

In the case of simplicial folding, the relations (5) and (6) of proposition 1.4 will take the following form respectively:

7) $g=1+\frac{k-6}{12} \alpha$, for orientable surfaces

8) $p=2+\frac{k-6}{6} \alpha$, for non orientable surfaces

Theorem 3.2 [3]: Any regular 3-folding of $S^{2}$ is equivalent to $\omega$.

Theorem 3.3: The sphere $S^{2}$ can be simplicially folded to $P_{3}$ if $\alpha, \beta$ and $\gamma$ of the graph $\Gamma_{f}$ satisfy the following relations:

$$
\alpha=2 i+4, \beta=6 i+6, \gamma=4 i+4 \text { where } i=1,2, \ldots
$$

In this case the class $O_{0}(3)$ is an infinite countable class.

Proof: Let $f_{m}: S^{2} \rightarrow P_{3}$ be the simplicial foldings of $S^{2}$ to $P_{3}$. From relations (1), (2) and (3) of proposition 1.4 , we have $3 \gamma=2 \beta, \alpha-\beta+\gamma=2$. Thus $\gamma=2 \alpha-4$, but $e(M) \leq \alpha\left(\frac{4}{3}-1\right), 2 \leq \frac{1}{3} \alpha$ and hence $\alpha \geq 6$.

Let $\alpha=6$, by using the above relations we get, $\gamma=8$ and $\beta=12$. In fact this folding is the octahedral folding $f_{1}=\omega$, see Fig. 2

Now, let $\alpha=7$, then $\gamma=10$ and $\beta=15$, i. e., the graph $\Gamma_{f}$ of this simplicial folding, if there exist any, has 7 vertices, 15 edges and 10 faces. This graph can be embedded in $S^{2}$ as shown in Fig. 3. 


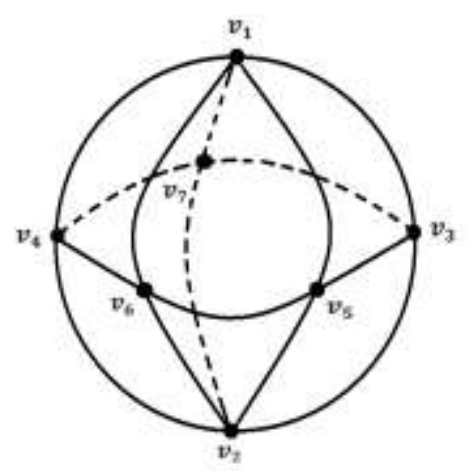

Fig. 3 Non folding graph of $S^{2}$ if $\alpha=7$

This graph is not a folding graph since each of the vertices $v_{1}$ and $v_{2}$ has an odd valency besides the graph does not be 3-colourable.

We know that $\sum_{v \in \Gamma_{f}} d(v)=2|E|$ and the valency of each vertex $\geq 4$ besides the number of vertices with odd valency is even. Generaly, if the number of vertices $\alpha$ is odd then there will exist at least two vertices of odd valency and hence in this case the graph cannot be a folding graph.

Now, let $\alpha=8$, then $\beta=18$ and $\gamma=12$. This graph can be embedded in $S^{2}$ as shown in Fig. 4

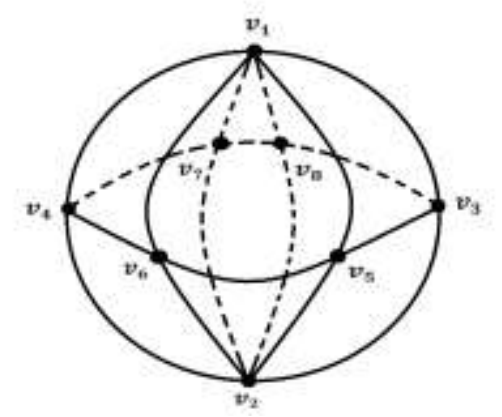

Fig. 4 Simplicial folding of $S^{2}$ if $\alpha=8$

This graph is 3 -colourable and each vertex has an even valency $\geq 4$. Thus the graph is a folding graph of a simplicial folding $f_{2}: S^{2} \rightarrow P_{3}$.

Consider $\alpha=9$, then $\gamma=14$ and $\beta=21$. The corresponding graph is not a folding graph since there is at least two vertices of odd valency. Fig. 5 shows an embedding of this graph in $S^{2}$.

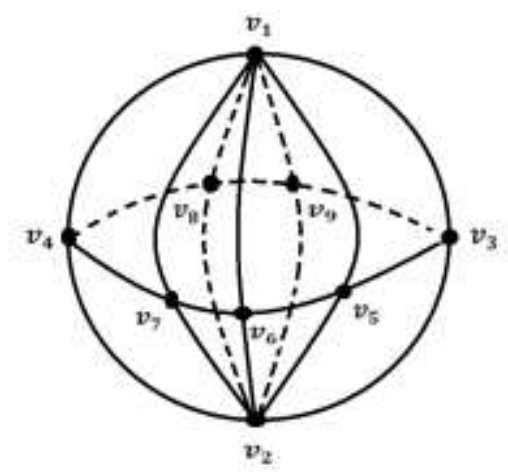

Fig. 5 Non folding graph of $S^{2}$ if $\alpha=9$ 
Now, let $\alpha=10$, then $\gamma=16$ and $\beta=24$. The corresponding graph can be embedded in $S^{2}$ as shown in Fig. 6 each vertex has even valency and the graph is 3-colourable. Thus, it represents a folding graph of a simplicial folding $f_{3}: S^{2} \rightarrow P_{3}$.

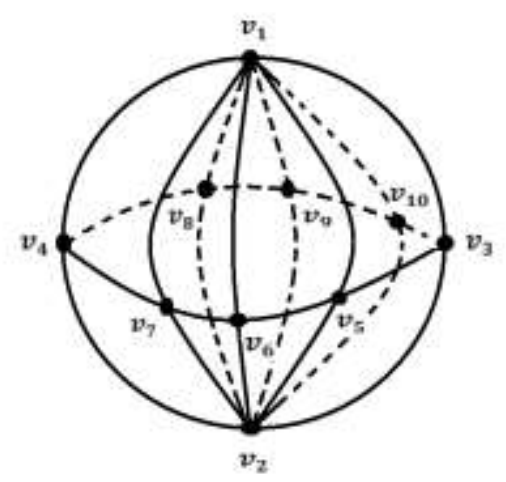

Fig. 6 Simplicial folding of $S^{2}$ if $\alpha=10$

In general, the sphere $S^{2}$ can be simplicially folded if $\alpha=2 i+4, \beta=6 i+6$ and $\gamma=4 i+4$, where $i=1,2, \ldots$, i. e., there exists an infinite countable number of simplicial folding of $S^{2}$ into $P_{3}$.

\subsection{Simplicial folding of the torus}

The torus is an orientable surface of genus 1 . In the following we will classify all the simplicial foldings of this surface.

Theorem 3.4: Any regular simplicial folding of the torus is of type $(6,3)$.

Proof: Let $f: T^{2} \rightarrow P_{3}$ be a regular simplicial folding of $T^{2}$ into $P_{3}$ of type $(k, 3)$, where $k$ is the valency of each vertex and $k \geq 4$. This graph has $\alpha$ vertices, $\beta$ edges and $\gamma$ faces. Then, from relation (7), where $g=1$, we get $1=1+\frac{k-6}{12} \alpha$. Thus, $(k-6) \alpha=0$ implies that $k=6$ and hence the regular simplicial folding is of type $(6,3)$. It should be noted that there are many other unequivalent regular simplicial foldings of $T^{2}$ into $P_{3}$ but all of type $(6,3)$, see Fig. 7 .
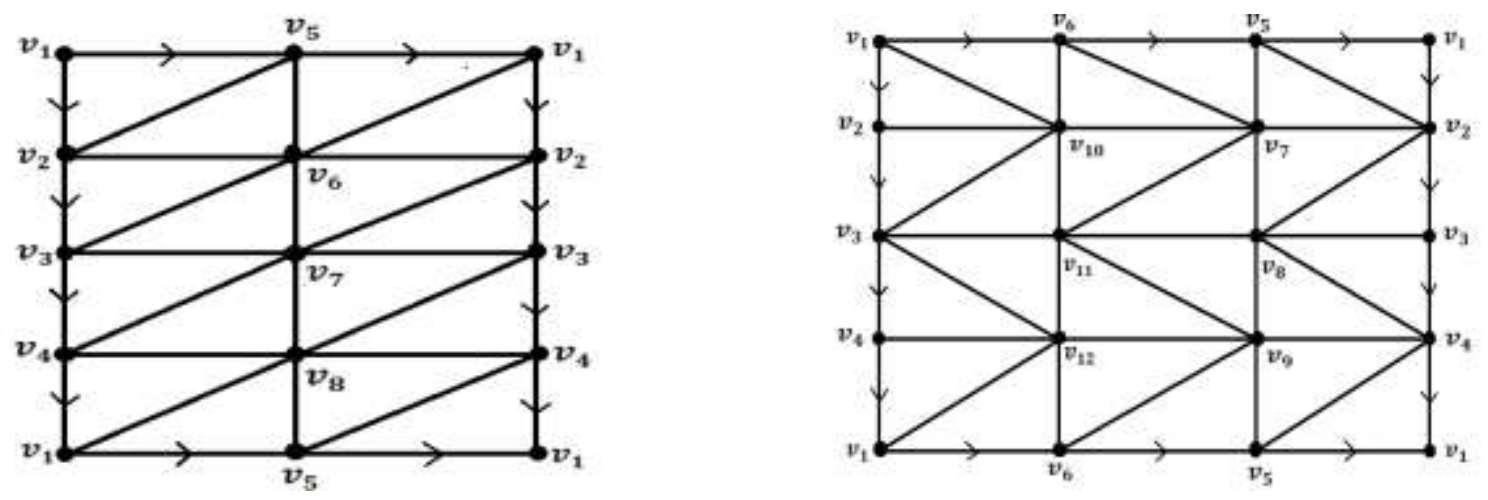

Fig. 7 Unequivalent regular foldings of type $(6,3)$ 
Theorem 3.5: The tours can be simplicialy folded if the numbers of vertices $\alpha$, edges $\beta$ and faces $\gamma$ of the folding graph satisfy the relations:

$$
\alpha=4 i+4, \beta=12 i+12 \text { and } \gamma=8 i+8, i=1,2, \ldots
$$

or the relation

$$
\alpha=9 i, \beta=27 i \text { and } \gamma=18 i, i=1,2, \ldots
$$

Thus $O_{1}(3)$ is an infinite countable class.

Proof: Suppose that $f_{m}: T^{2} \rightarrow P_{3}$ are the simplicial foldings of $T^{2}$. From relations (1), (2) and (3) of Proposition 1.4 we get, $3 \gamma=2 \beta$ and $2 \beta-2 \gamma=2 \alpha$. Thus $\gamma=2 \alpha$, also $e(M) \leq \alpha\left(\frac{4}{3}-1\right)=\frac{\alpha}{3}$ i.e., $\alpha \geq 0$.

But the minimum number of vertices $\alpha$ needed to triangulate the torus satisfy the relation $\alpha \geq \frac{1}{2}(7+$ $\sqrt{49-24 e(M)})=7$, see [7].

If $\alpha=7$, then $\gamma=14$ and $\beta=21$. This graph can be embedded in $T^{2}$ as a regular graph of valency 6 as shown in Fig. 8 but this graph is not 3-colourable and hence is not a folding graph.

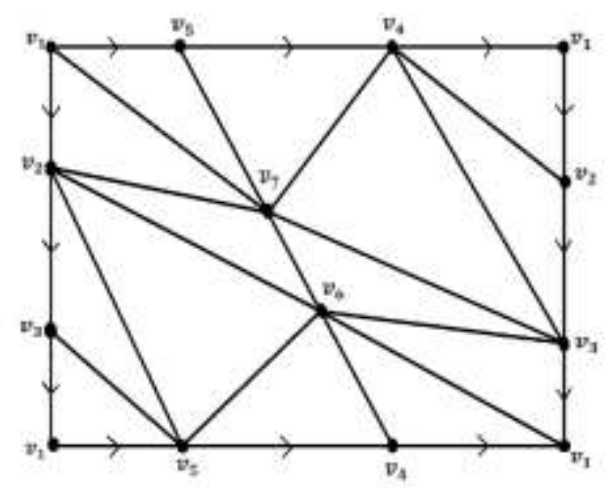

Fig. 8 Non folding graph of $T^{2}$ if $\alpha=7$.

Let $\alpha=8$, then $\gamma=16$ and $\beta=24$. This graph can be embedded in $T^{2}$ in different ways some is colourable and the others is not, Fig. 9 shows two of them. Thus we can define a simplicial folding $f_{1}: T^{2} \rightarrow$ $P_{3}$

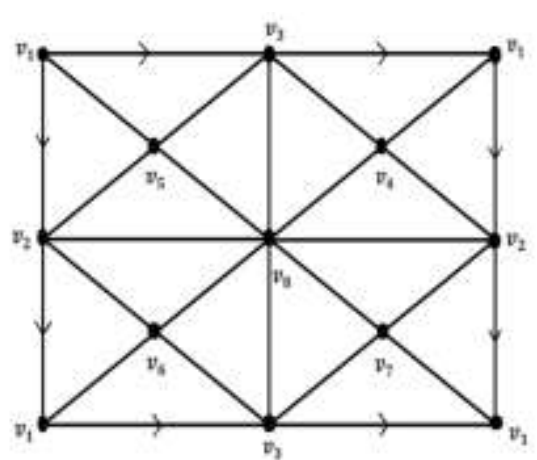

Fig. 9 (a): Folding graph with $\alpha=8$

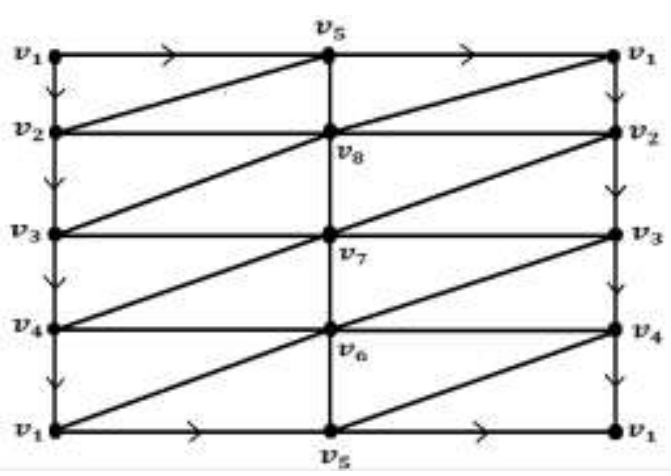

Fig. 9 (b): Non folding graph with $\alpha=8$

Now, let $\alpha=9$, then $\gamma=18$ and $\beta=27$. This graph can be embedded in $T^{2}$ as a regular graph of type (6, 3) which is 3-colourable. Thus we can define a simplicial folding $f_{2}: T^{2} \rightarrow P_{3}$, see Fig. 10. 


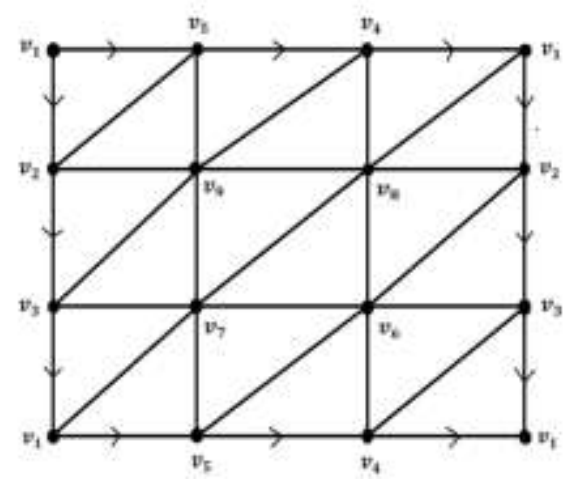

Fig. 10 Folding graph with $\alpha=9$

If $\alpha=10$, then $\gamma=20, \beta=30$. This graph can be embedded in $T^{2}$ in different ways.

Fig. 11 shows some of them, (a) represents two regular graphs of valency 6 and (b) represents two irregular graphs. All these graphs are not 3-colourable and hence they do not represent folding graphs of any simplicial folding of $T^{2}$.
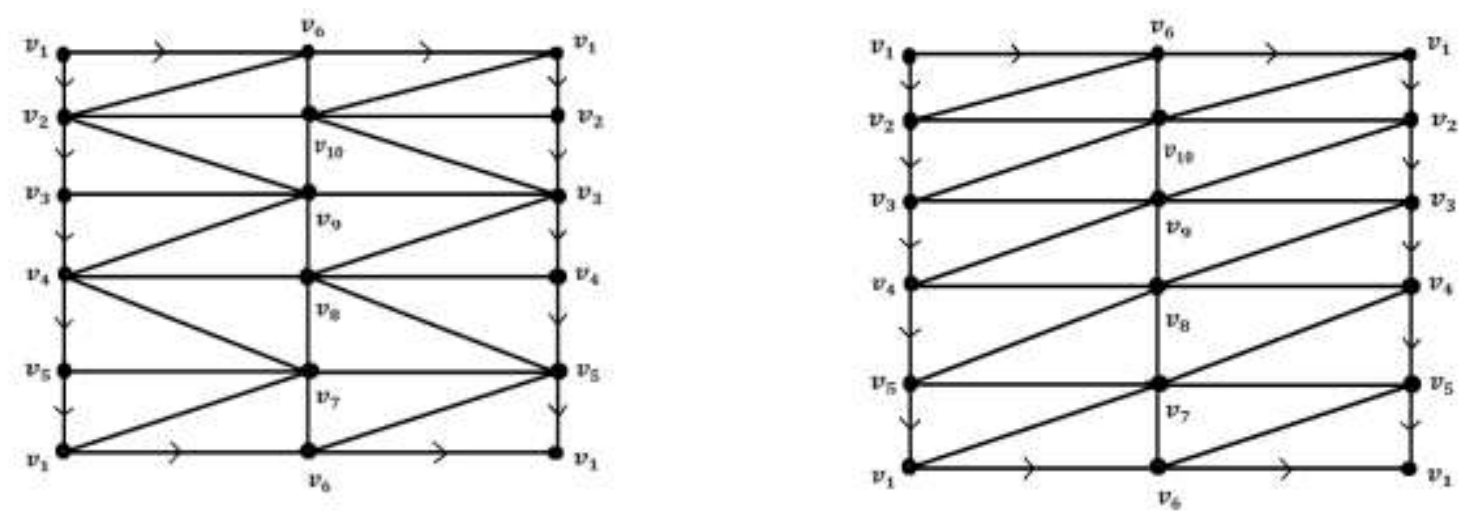

Fig. 11 (a): Regular graphs with $\alpha=10$, but not 3-colourable
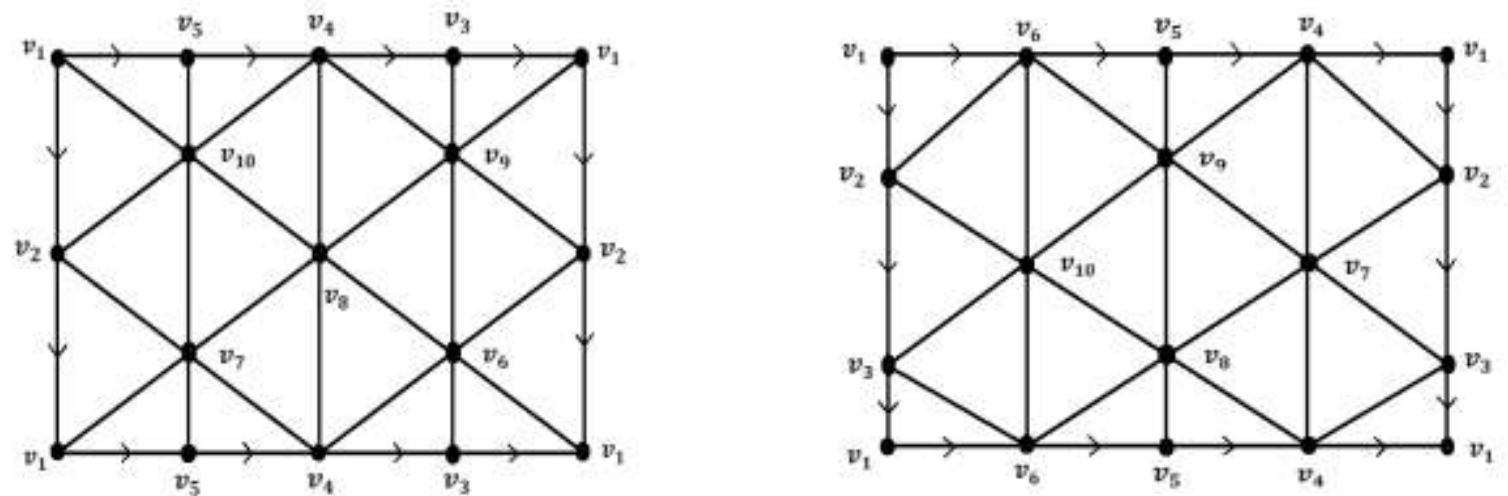

Fig. 11 (b): Irregular graphs with $\alpha=10$, but not 3-colourable

By using the same procedure we can easily check that the possibilities of simplicially folding $T^{2}$ are given by:

$$
\alpha=4 i+4, \beta=12 i+12 \text { and } \gamma=8 i+8, i=1,2, \ldots
$$

or

$$
\alpha=9 i, \beta=27 i \text { and } \gamma=18 i, i=1,2, \ldots
$$

Thus the class $O_{1}(3)$ is a countable infinite class. 
Fig. 12 shows 3 -colourable folding graphs embedded in $T^{2}$ corresponding to $\alpha=12$ and $\alpha=16$, i. e. satisfied relations (i) while Fig. 13 shows 3 -colourable folding graphs embedded in $T^{2}$ corresponding to $\alpha=18$ and $\alpha=27$, i.e. satisfied relations (ii).

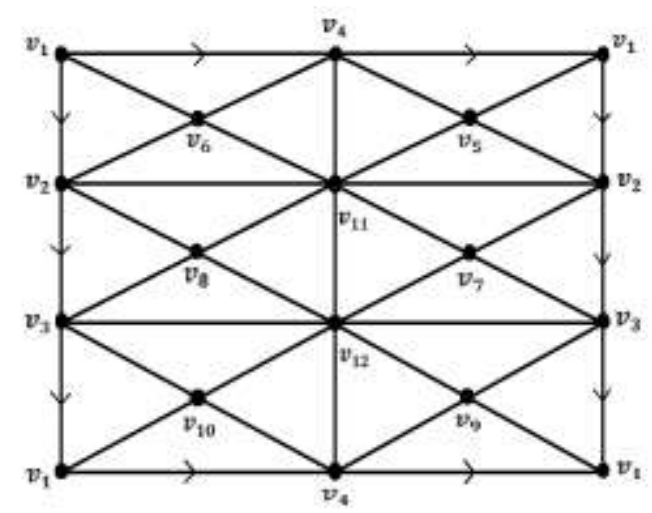

Fig. 12 (a): $\alpha=12$

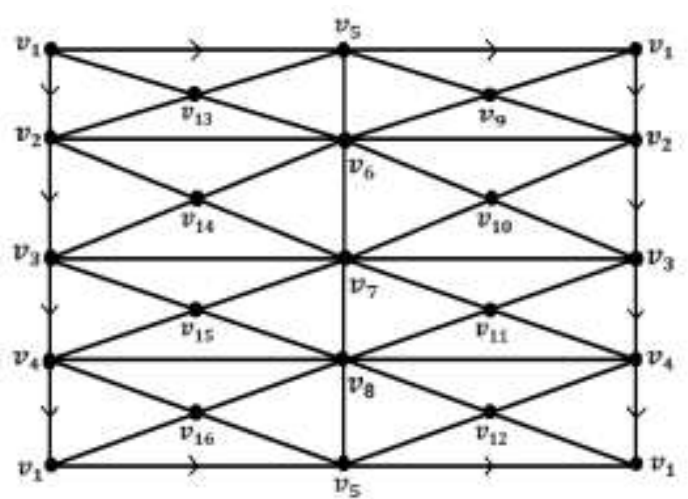

Fig. 12 (b): $\alpha=16$

3-colourable folding graphs of $T^{2}$ corresponding $\alpha=12, \alpha=16$

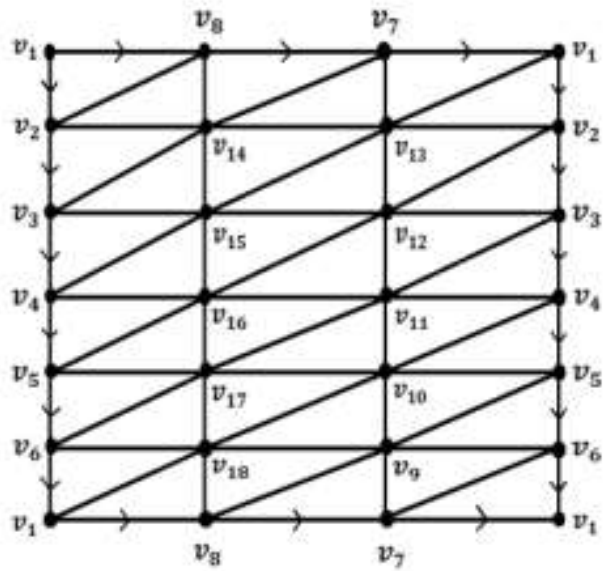

Fig. 13 (a): $\alpha=18$

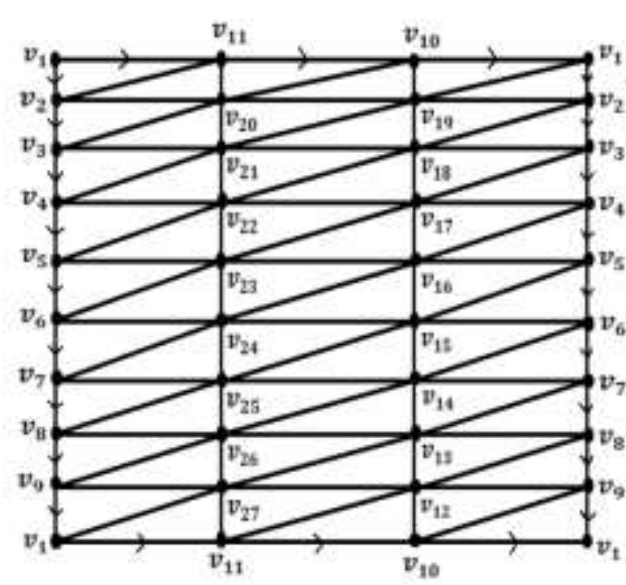

Fig. 13 (b): $\alpha=27$

3-colourable folding graphs of $T^{2}$ corresponding $\alpha=18, \alpha=27$

\subsection{Simplicial folding of the connected sum of tori}

We will start with $T_{1}^{2} \# T_{2}^{2}$, i.e., the double torus which is an orientable surface of genus 2 .

Theorem 3.6: There is no regular simplicial folding of the double torus to $P_{3}$.

Proof: From relation (7), $g=2$, we get

$$
2=1+\frac{k-6}{12} \alpha \text {. Thus } \alpha=\frac{12}{k-6}, \text { but } \alpha>0 \text {. }
$$

This implies that $k>6$. Also if $k>10$, then $\alpha \leq 2$ and this is refused and consequently $k \leq 10$, i.e., $6<k \leq 10$. This means that $k=8$ or 10 .

If $k=8$, then $\alpha=6$, but the minimum nmber of the vertices $\alpha$ to triangulate the double torus is

$$
\alpha \geq \frac{1}{2}(7+\sqrt{49-24 e(M)})=\frac{1}{2}(7+\sqrt{49+48}),
$$

i.e. $\alpha \geq 8$, and hence we cannot triangulate the double torus if $\alpha=6$.

Now, if $k=10$, then $\alpha=3$. Once again, we cannot triangulate the double tours in this case. Hence, this surface cannot regularly simplicially folded. 
Theorem 3.7: The double tours can be simplicially folded an infinite time, i.e., the class $O_{2}(3)$ is an infinite countable class.

Proof: Suppose each of $M$ and $N$ is a tours and let $f: M \rightarrow P_{3}, g: N \rightarrow P_{3}$ be simplicial foldings. Choose two 2-simplexes, triangles, $A$ and $B$ of $M$ and $N$ respectively. Let $u_{1}, u_{2}, u_{3}$ be the vertices of $A$ and $v_{1}, v_{2}, v_{3}$ be the vertices of $B$ such that $f\left(u_{i}\right)=g\left(v_{i}\right)$.

Now, remove the interior of $A$ and $B$ from $M$ and $N$ respectively and identify the resulting surfaces along the boundaries of $A$ and $B$ in such a way that $u_{i}$ and $v_{i}$ are identified. This gives a simplicial folding of $M \# N$, i.e., the double torus, because the resulting new folding graph $\Gamma_{f \# g}$ is also 3-colourable with the coloring inherited from those of $\Gamma_{f}$ and $\Gamma_{g}$.

By using theorem 3.5 we can calculate the cases for which there exists a simplicial folding of the double tours. These cases are of cause infinite, i.e. $O_{2}(3)$ is an infinite class.

Note that by using the same procedure, we can show that the connected sum of $g$ tori can be simplicially folded. In fact $O_{g}(3)$ is infinite.

\section{SIMPLICIAL FOLDING OF NON-ORIENTABLE SURFACES}

In the following, we classify all simplicial folding of the projective plane $P^{2}$ and the connected sum of projective planes.

\subsection{Simplicial folding of the projective plane $P^{2}$}

The projective plane $P^{2}$ is a non-orientable 2-manifold of genus 1 . Now, we will classify all the possible simplicial foldings of $P^{2}$.

Theorem 4.1: There is no regular simplicial folding of the projective plane.

Proof: From relation $(8), p=1$, we get

$$
1=2+\frac{k-6}{6} \alpha \text {. Thus } \alpha=\frac{6}{6-k},
$$

But the number of the vertices $\alpha>0$ and hence $k<6$. Also $k \geq 4$, i.e., $4 \leq k<6$ and hence $k=4$. By using the relation $\alpha=\frac{6}{6-k}$, we get $\alpha=3$. Now the minimum number of vertices needed to triangulate the projective plane is $\left[\sqrt{12-6 e(M)+\frac{5}{2}}\right]+2$, for $e(M) \neq 0,-1$, i.e. $\alpha \geq 6$, [7]. Thus there is no regular simplicial folding of $P^{2}$.

Theorem 4.2: The projective plane can be simplicially folded if $\alpha, \beta$ and $\gamma$ satisfying the following relations:

$$
\alpha=i+2, \beta=3 i+3 \text { and } \gamma=2 i+2, i=1,2, \ldots
$$

Proof: Consider the projective plane $P^{2}$, but the sphere $S^{2}$ is a double covering of $P^{2}$, where $\theta: S^{2} \rightarrow P^{2}$ is the projection map. Thus if $f: P^{2} \rightarrow P_{3}$ is a simplicial folding of $P^{2}$, then $f \circ \theta: S^{2} \rightarrow P_{3}$ is also a simplicial folding. Conversely, if $g: S^{2} \rightarrow P_{3}$ is a simplicial folding, this folding by using the projection map $\theta$ will induce a simplicial folding of the projective plane [3].

This means that simplicial foldings of the sphere induce simplicial foldings of the projective plane for which the number of vertices, edges and faces of the folding graph of $S^{2}$ is half of that of the sphere, but by theorem 3.3 the sphere can be simplicially folded if the vertices, edges and faces of the its folding graph satisfy the relations

$$
\alpha=2 i+4, \beta=6 i+6 \text { and } \gamma=4 i+4, i=1,2, \ldots
$$

Thus the vertices, edges and faces of the folding graph of the projective plane must satisfy the relations $\alpha=i+2, \beta=3 i+3$ and $\gamma=2 i+2, i=1,2, \ldots$. 


\subsection{Simplicial folding of the connected sum of projective planes}

Consider first the connected sum of two projective planes $P_{1}^{2} \# P_{2}^{2}$. This surface is a non-orientable surface of genus 2 .

Theorem 4.3: Any regular simplicial folding of $P_{1}^{2} \# P_{2}^{2}$ is of the type $(6,3)$.

Proof: Let $f: P_{1}^{2} \# P_{2}^{2} \rightarrow P_{3}$ be a regular simplicial folding of $P_{1}^{2} \# P_{2}^{2}$ into $P_{3}$ of type $(k, 3)$, where $k \geq 4$ is the valency of each vertex. From relation (7), where $p=2$, we get $2=2+\frac{k-6}{6} \alpha$. Thus, $(k-6) \alpha=0$, this implies that $k=6$ and hence the regular simplicial folding is of type $(6,3)$.

Theorem 4.4: The connected sum of $p$ projective planes can be simplicially folded an infinite number.

Proof: Suppose that $N_{p}=\#^{p} P^{2}$ is the connected sum of $p$ projective planes. For any surface $N_{p}$ there exist a double covering $\theta_{p}: M_{g-1} \rightarrow N_{p}$, where $M_{g}=\#^{g} T^{2}, p>1$, the double cover $\theta_{1}: M_{0}=S^{2} \rightarrow N_{1}=P^{2}$ is the double cover of the projective plane.

Once again if $f: N_{p} \rightarrow P_{3}$ is a simplicial folding, the $\theta_{p} \circ f$ is also a simplicial folding and the converse is true, by using the simplicial foldings of the tori we can obtain an infinite number foldings of the connected sum of the projective planes.

\section{CONCLUSION AND REMARKS}

Our main interest in this paper is to know whether and how many cellular foldings of a given surface onto a given polygon do exist and to classify regular simplicial and simplicial foldings of orientable and nonorientable surfaces.

Now, we put our hands on an open problem. Let $f: M \rightarrow P_{n}$ be a regular folding and let $C_{f}$ be the cell decomposition of $M$ associated to $f$. Let $H(f)$ be the set of homeomorphisms $h: M \rightarrow M$ which are also cellular maps of $C_{f}$ at the same time. $H(f)$ becomes a group with respect to the composition of homeomorphisms.

The problem is to know the action of $H(f)$ on the cells of $C_{f}$. A typical question is whether $H(f)$ acts transitively on the 2-cells of $C_{f}$.

\section{REFERENCES}

[1] E. EL-KHOLY AND R. M. SHAHIN, Cellular foldings, J. Inst. Math. and Comp. Sci. (Math. Ser.), India, vol.11, No. 3. pp. 177-181 (1998).

[2] FARRAN, H. R., EL-KHOLY, E. AND ROBERTSON, S. A.; Folding of star manifolds, Proc. Roy. Soc. Edinburgh Sect. A123, No. 6, 1011-1016, (1993).

[3] H. R. FARRAN, E. EL-KHOLY AND S. A. ROBERTSON, Folding a surface to a polygon, Geometriae Dedicata 63, 255-266, 1996, Germany

[4] R. M. SHAHIN; Regular folding of surfaces, Ph. D. Thesis, Faculty of science, Tanta University (1999).

[5] E. EL-KHOLY AND M. EL-GHOUL, Simplicial folding, Jour. Fac. Education, No. 7, Part 11, pp. 127-139, (1984).

[6] E. EL-KHOLY, A. EL-ESAWY AND M. A. KOUKA, cellular folding of some new CWcomplexes, Bull. Math.\& Statistics Research, vol. 4, Issue 4, 1-12, (2016).

[7] P. J. GIBLIN, Graphs, Surfaces and Homology groups, Chapman and Hall, 1977, London, U. K. 